\title{
Healthy Learning Mind - a school-based mindfulness and relaxation program: a study protocol for a cluster randomized controlled trial
}

Salla-maarit Volanen ${ }^{1,2^{*}}$ (D), Maarit Lassander ${ }^{3}$, Nelli Hankonen ${ }^{4}$, Päivi Santalahti ${ }^{5}$, Mirka Hintsanen ${ }^{6}$, Nina Simonsen ${ }^{1,2}$, Anu Raevuori ${ }^{2,7,8}$, Sari Mullola ${ }^{3,9}$, Tero Vahlberg ${ }^{10}$, Anna But ${ }^{2}$ and Sakari Suominen ${ }^{1,11,12}$

\begin{abstract}
Background: Mindfulness has shown positive effects on mental health, mental capacity and well-being among adult population. Among children and adolescents, previous research on the effectiveness of mindfulness interventions on health and well-being has shown promising results, but studies with methodologically sound designs have been called for. Few intervention studies in this population have compared the effectiveness of mindfulness programs to alternative intervention programs with adequate sample sizes.

Methods/design: Our primary aim is to explore the effectiveness of a school-based mindfulness intervention program compared to a standard relaxation program among a non-clinical children and adolescent sample, and a non-treatment control group in school context. In this study, we systematically examine the effects of mindfulness intervention on mental well-being (primary outcomes being resilience; existence/absence of depressive symptoms; experienced psychological strengths and difficulties), cognitive functions, psychophysiological responses, academic achievements, and motivational determinants of practicing mindfulness.

The design is a cluster randomized controlled trial with three arms (mindfulness intervention group, active control group, non-treatment group) and the sample includes 59 Finnish schools and approx. 3000 students aged 12-15 years. Intervention consists of nine mindfulness based lessons, 45 mins per week, for 9 weeks, the dose being identical in active control group receiving standard relaxation program called Relax. The programs are delivered by 14 educated facilitators. Students, their teachers and parents will fill-in the research questionnaires before and after the intervention, and they will all be followed up 6 months after baseline. Additionally, students will be followed 12 months after baseline. For longer follow-up, consent to linking the data to the main health registers has been asked from students and their parents.
\end{abstract}

Discussion: The present study examines systematically the effectiveness of a school-based mindfulness program compared to a standard relaxation program, and a non-treatment control group. A strength of the current study lies in its methodologically rigorous, randomized controlled study design, which allows novel evidence on the effectiveness of mindfulness over and above a standard relaxation program.

Trial registration: ISRCTN18642659. Retrospectively registered 13 October 2015.

Keywords: Children and adolescents, School-based intervention, Mindfulness, Health promotion, Mental health, Well-being

\footnotetext{
* Correspondence: salla-maarit.volanen@helsinki.fi

${ }^{1}$ Folkhälsan Research Center, Topeliuksenkatu 20, 00250 Helsinki, Finland

${ }^{2}$ Department of Public Health, University of Helsinki, Helsinki, Finland

Full list of author information is available at the end of the article
} 


\section{Background}

In the contemporary society, children and adolescents have to deal with several stressors on daily basis. Stressors may arise from family-system disturbances, peer conflicts, school context, socio-cultural challenges, vulnerabilities to physical and mental health problems, or from living in the fast-paced, media-saturated and multi-tasking world that sets high demands for performance, success and competition [1]. Research suggests that sustained stress in childhood and adolescence has negative influence on mental health, general functioning, and specific learning-related factors, such as executive function and working memory [2]. Approximately one fourth of youth suffers from at least one mental disorder during the past year, and respectively, about one third suffers from any lifetime mental disorder. Anxiety disorders are the most frequent mental disorders in children and adolescents, followed by behavior disorders, the mood disorders and substance use disorders [3]. In Finland, approximately $14 \%$ of children aged eight to nine years suffer from some kind of mental health problems, and this share steeply arises along with the onset of puberty to $15-25 \%$ in adolescent population [4]. Psychiatric disorders are the most important disorder group that impairs adolescents' functional ability [5], and perceived stress is shown to increase the risk of subsequent mental disorders and their symptoms [6-8]. Thus, there is a need for effective, disseminable strategies to protect children and youth from dysfunctional effects of stress.

During the last few years, research on mindfulness has increased, and extended from initially focusing only on adults to including children and adolescents as well. However, studies with methodologically sound designs are still lacking. To be able to indicate the significant beneficial effects of mindfulness practice also on children's and adolescents' health and well-being, research needs to shift toward large, well-designed studies with robust methodologies, and adopt standardized formats of interventions, allowing for replication and comparison of studies, to develop a firm evidence base [9].

\section{Mindfulness and health}

Mindfulness refers to a non-condemning state of awareness and readiness to pay attention to the stream of experiences in the present moment [10]. The concept is rooted in Eastern contemplative traditions and was later developed as part of therapeutic applications in psychology and medicine, such as mindfulness-based stress reduction (MBSR) [10, 11], mindfulness-based cognitive therapy [12], dialectic behavior therapy [13], and acceptance and commitment therapy (ACT) $[14,15]$. The beneficial elements of mindfulness are suggested to include e.g. attention regulation, body awareness, emotion regulation, and change in perspectives on the self and learning [16]. Research among adults has shown that mindfulness practices reduce negative states of mind, such as stress [17], and symptoms of anxiety and depression [18-20], as well as alleviate various medical conditions, such as chronic pain [10] , type 2 diabetes [21, 22] and attention-deficit hyperactivity disorder [23, 24].

Furthermore, research among adults has shown promising positive associations between mindfulness practice and health behaviours, such as smoking cessation [25, 26], decreased binge eating [27], and decreased alcohol and substance use [28]. Finally, practicing mindfulness has also been shown to produce positive effects on psychological well-being in healthy participants [29-31].

Recently, also brain imaging has been utilized to study the neural level effects related to mindfulness based practices or meditation. Changes are reported both in structural properties $[32,33]$ and in brain functioning $[19,34]$, especially related to attentional control [35] and emotion regulation [36, 37].

In the previous decade, interest started to spread to mindfulness based approaches with children and adolescents and international research has shown promising preliminary results both in clinical context [23, 38-42] and in non-clinical, school context [43-49].

\section{Mindfulness among children and adolescents in school setting}

It has been reported that mindfulness interventions are acceptable for children and adolescents, as well as feasible, and that they improve for example attention, emotional reactivity and some areas of meta-cognition [1]. Mindfulness-based programs have improved school-aged children's attention and teacher-rated social skills [45]. A school-based (RCT) study showed significant improvements in post-treatment measures of self-rated test anxiety, teacher rated attention, social skills, objective measures of selective (visual) attention but no sustained attention, as well as improved behavioral regulation, metacognition, and overall global executive control among children who started out with poor executive functions [43]. Correspondingly, in another study [44] adolescents with lower pre-intervention self-regulation were observed to experience greatest improvements in behavioral regulation, meta-cognition and executive function. Preliminary research has shown that school-based mindfulness intervention programs may also result in beneficial outcomes regarding the interaction and pedagogical atmosphere among both students and students and their teachers [50].

In the school setting, mindfulness interventions reach the whole age group, and through the equal reach may even act as a counterforce for the prominent development of increasing inequality between different groups (based on e.g. gender, learning difficulties, health challenges, or socioeconomic background), yet empirical evidence is lacking. 
While cost-effectiveness and ease of implementation of mindfulness programs in schools are notable advantages, sufficient evidence is still lacking on the role of mindfulness in fostering resilience, mental health and well-being among children and adolescents, over and above existing approaches such as relaxation. The previous studies conducted among youth are still few in number $[1,9,23,38-40,43-45,51,52]$, and their methodological shortcomings (e.g. small sample sizes without control groups and/or unstandardized mindfulness intervention programs) prevent making generalizations of the efficacy of these interventions [9]. For instance, it is not well understood whether the observed changes persist or what the short and long-term effects of mindfulness intervention are [40]. Further, the role of mindfulness in improving health behavior among adolescents is not well known [53, 54].

It might be at place to state here also that the Finnish school system offers exceptionally good possibilities for examining between-individual variation as the schoolrelated variance is minimized due to the homogenous schools system of our country: All schools follow the national curriculum, private schools are almost non-existent, and majority of students go to the nearest school in their residential area. Also areal segregation is still rather low compared to other countries. Furthermore, all teachers receive university education which reduces the teacherrelated variance.

\section{The aim of the study}

The comprehensive aim of this ongoing trial is to examine the effects of mindfulness practices in strengthening children's and adolescents' internal resources that promote mental wellbeing, cognitive functions, psycho-physiological responses, academic achievement, health behavior, motivational determinants of practice compared to a standard relaxation program and a non-treatment group (waitinglist). The primary aim is to determine the effectiveness of the school-based mindfulness program on three main outcomes: resilience (RS14), existence or absence of depressive symptoms (RBDI), and experienced psychological strengths and difficulties (SDQ). Secondary outcomes include mindfulness, happiness, satisfaction with life, quality of life, positive and negative affects, compassion/self-kindness, the rumination, and stress. Other explored factors among children and adolescents are cognitive functions, psychophysiological responses, academic achievement, health behavior, motivational determinants of practicing mindfulness, and class room social environment. The study will also explore equity of distribution of the primary outcomes in terms of social background, gender, and learning difficulties of the students. The results of the study will be presented according to the 2010 CONSORT statement [55] and its extension to cluster randomized controlled trials [56].

\section{Methods}

\section{Trial design}

The study is an ongoing cluster randomized controlled trial (RCT) with three arms. Eligible schools were randomly allocated either to an intervention, control or nontreatment groups. Clusters were school classes (grades 6, 7 and 8) and age gap was from 12 to 15 years olds. The data collection started in the spring 2014, and finishes in the autumn 2016. The analyzing and reporting of the data starts in the autumn 2016.

\section{Randomization procedure}

The recruitment started by listing all the schools in a Southern part of Finland. After choosing the schools (including as many classes of the same grade as possible), a letter explaining the study procedure was sent by e-mail to the head masters. Within few days after sending the information letter, the research team members called the headmasters by telephone. In most schools the decision to take part to the study was made collectively by the head master and the class teachers (of the chosen grades). The schools were enrolled from 14 cities/municipalities during the collection of the data (years 2014-2016). Altogether 247 schools were contacted, 59 of those participated in the study participation percentage being 24 . In each municipality we aimed at an equal number of intervention and control classes. In order to achieve balanced intervention and control groups, schools participating in the study were randomized using the available background variables. The selection of intervention-control pairs was primarily based on the language being used for teaching (Finnish, Swedish or English, the grade, the school location, the number of classes participating in the investigation and, if necessary, the average apartment price per square meter in the school's neighborhood).

The classes were randomly assigned to mindfulness intervention classes $(N=85)$ and control classes $(N=79)$ and non-treatment classes $(N=28)$. Due to practical reasons, in spring 2014 and in autumn 2015 schools were divided into two arms (intervention and control) and in spring 2015 and spring 2016 into three arms instead of two: intervention, control and non-treatment groups. First, the schools were divided into three groups based on the school location and the average apartment price per square meter. Within each of these groups, the total number of schools and classes varied. Next, the schools for these groups were divided into three subgroups including approximately same number of classes (some schools were combined into one subgroup to achieve an as even distribution of classes as possible).

\section{Data collection timeline}

The data from intervention and control groups have been collected during four academic terms: In the beginning of 
spring term in $2014(N=523)$, in the beginning of autumn term in $2014(N=1090)$, in the beginning of spring term in $2015(N=821)$, and in the beginning of spring term in 2016 (on going, baseline including $N=203$ ). Hence the last follow-up will be collected in spring 2017 (12 months follow-up of the spring 2016). Among intervention and control groups data have been collected at baseline, in the middle of the intervention (the fifth week of the intervention, a short formula), within 1 week after the intervention, and 6 and 12 months after baseline from the same participants.

Due to practical reasons, the data from non-treatment group have been collected during two academic terms: In the beginning of spring term in $2015(N=254)$, and in the beginning of spring term 2016 (ongoing, baseline including $N=109$ ). Additionally, non-treatment group did not fill in the short formula in the middle of the intervention the measurement points being otherwise identical with the other two groups (incl. follow-ups).

Among teachers and parents data have been collected at baseline, after the intervention and 6 months after the baseline from the same parent (if only one parent filledin the formula) and from the same teacher. In a case the teacher had left/changed between the different measurement points, only the grades and absence from school of students were asked (from the new teacher).

\section{Measurements \\ Students \\ Questionnaire}

A comprehensive set of standardized questionnaires is being filled in by all participants (Table 1).

Students fill in their questionnaires at school under facilitators' or teachers' monitoring. Parents fill in their questionnaires at home and bring/send them to school in a closed envelope. Teachers fill in their questionnaires during their working hours at school, if possible. The filled questionnaires (students, teachers, parents) are collected from schools and brought to recording company's premises approximately $2-3$ weeks after the intervention period has finished.

Primary outcomes In children's and adolescents, existence or absence of depressive symptoms was measured with the Finnish version of the Beck Depression Inventory (RBDI) [57]. The well-being was measured with the Strenghts and Difficulties Questionnaire (SDQ) [58]. The resilience was measured with Resilience Scale (RS14) [59] that has shown good internal consistency reliability among adults, Cronbach Alpha (CA) 0.87 [60]. The Finnish versions of SDQ [61], Cronbach Alpha (CA) 0.71 and RBDI $[62,63]$ CA $0.83,0.87$ have shown adequate psychometric properties among youth.
Secondary outcomes The secondary outcomes of the present study are conceptualized as children's and adolescents' cognitive-emotional factors that are essential for their resilience, mental health and well-being; Mindfulnesss, Happiness, Satisfaction with Life, Quality of Life, Positive and Negative affects, and compassion/self-kindness, the rumination, and stress. Additionally cognitive functions, psychophysiological responses, academic achievements, health behavior, and motivational determinants of practice have been included in the present study (Table 1).

Psycho-physiological and neuropsychological measures Both the objective neuropsychological and psychophysiological measures were collected from a subset of students: 62 students in the intervention group and 69 students in the control group (relaxation programme) were randomly selected from four 6th grade and four 8 th grade classes $(N=131)$. There were three measurement points: before the intervention started, directly after the intervention period, and 6 months after the intervention period. Neuropsychological tests include subtests from NEPSY-II [64], WISC-IV [65] and D-KEFS [66].

NEPSY-II [64] (Developmental Neuropsychological Assessment) is a series of neuropsychological tests, used in various combinations to assess neuropsychological development in children [64]. In this study we will administer the test of Inhibition, measuring the ability to inhibit and switch response types, which is a part of the attention and executive functioning domain category.

WISC-IV (Wechsler Intelligence Scale for Children) is a well-known and widely used assessment of cognitive functioning in children [65]. We administer the Working memory subtest, which assesses the ability to hold and manipulate new information in the short-term memory.

$D$-KEFS (Delis-Kaplan Executive Function System) is set of neuropsychological tests used to measure variety of verbal and non-verbal executive functions [66]. The subtests to be administered include the Trailmaking test (measuring flexibility of thinking on a visual-motor sequencing task) and the Verbal fluency test (measuring letter, category and category switching fluency).

Psycho-physiological measures The psycho-physiological measurement will be conducted with the mobile Nexus instruments from the psychology laboratory in Helsinki University. The measurement includes skin conductance response, heart rate and electrocardiography.

Skin conductance response [67] method for measuring the electrical conductance of the skin which varies with moisture level. Sweat glands are controlled by the sympathetic nervous system, so skin conductance is used as an indication of psychological or physiological arousal. Therefore, if the sympathetic branch of the autonomic nervous system is highly aroused, sweat gland activity will also 
Table 1 Outcome measures

\begin{tabular}{|c|c|c|c|c|}
\hline \multirow[t]{2}{*}{ Outcomes } & \multicolumn{3}{|c|}{ Informant } & \multirow[t]{2}{*}{ Measurement } \\
\hline & Student & Teacher & Parent & \\
\hline \multicolumn{5}{|l|}{ Mental Wellbeing } \\
\hline \multicolumn{5}{|l|}{ Primary outcomes } \\
\hline Resilience, resilience scala (RS14) & $x$ & & & \\
\hline Existence or absence of depressive symptoms (RBDI) & $x$ & & & \\
\hline Experienced psychological strenghts and difficulties (SDQ) & $x$ & $x$ & $x$ & \\
\hline \multicolumn{5}{|l|}{ Secondary outcomes } \\
\hline Mindfulness (CAMM) & $x$ & & & \\
\hline Happiness (OECD Better life Index) & $x$ & & & \\
\hline Satisfaction with life (SWLS-C) & $x$ & & & \\
\hline Quality of life (KINDL-R) & $x$ & & $x$ & \\
\hline Positive and negative affects (PANAS) & $x$ & & & \\
\hline \multicolumn{5}{|l|}{ The Rumination-Reflection Questionnaire } \\
\hline \multicolumn{5}{|l|}{ Stress in Children (SIC Qestionnaire) } \\
\hline Compassion/self-kindness) & $x$ & & & \\
\hline \multicolumn{5}{|l|}{ Cognitive measuments } \\
\hline NEPSY-II (Developmental Neuropsychological Assesment) & & & & $x$ \\
\hline WISC-IV (Wechsler Intelligence Scale for Children) & & & & $x$ \\
\hline D-KEFS (Delis-Kaplan Executive Function System) & & & & $x$ \\
\hline Psychological flexibility (CERQ) & $x$ & & & \\
\hline Viivi, 5-15 questionnaire on child development & & & $x$ & \\
\hline \multicolumn{5}{|l|}{ Psycho-physiological responses } \\
\hline SCR (Skin conduct response) & & & & $x$ \\
\hline EKG (Electrocardiography) & & & & $x$ \\
\hline \multicolumn{5}{|l|}{ Academic achievement/school } \\
\hline Grade average in the last school report & $x$ & $x$ & & \\
\hline Grades in last school report & $x$ & $x$ & & \\
\hline Satisfaction with ow achievements & $x$ & & & \\
\hline Days of absence from school & & $x$ & & \\
\hline Bullying at school & $x$ & & & \\
\hline \multicolumn{5}{|l|}{ Health behavior in school-aged children, WHO HBSC } \\
\hline Physical activity & $x$ & & & \\
\hline Sleeping/tiredness & $x$ & & & \\
\hline Alcohol use & $x$ & & & \\
\hline Smoking & $x$ & & & \\
\hline Screen time & $x$ & & & \\
\hline \multicolumn{5}{|l|}{ Motivational determinants of practice } \\
\hline Outcome expectations & $x$ & & & \\
\hline Use of strategies to relax & $x$ & & & \\
\hline Self-efficacy & $x$ & & & \\
\hline Intention/motivation & $x$ & & & \\
\hline Class room social environment (CES) & $x$ & $x$ & & \\
\hline Personality inventory (TIPI) & $x$ & & & \\
\hline
\end{tabular}


Table 1 Outcome measures (Continued)

\author{
Psycho-social background factors \\ Experienced major difficulties in life \\ The quality of social relationship with peers \\ Major changes in student's life \\ The emotional athmospere at home \\ The relationship with mother \\ The relationship with father \\ Socio-demopraphic background factors \\ Financial situation in the family \\ Family composition \\ Mother tongue \\ Parent education \\ occupation \\ Employment status
}$$
x
$$

$x$$$
x
$$$$
x
$$

$x$ increase, which in turn increases skin conductance. In this way, skin conductance can be used as a measure of emotional and sympathetic responses. A pair of electrodes is attached to palm or fingers to measure the response over a period of time.

Electrocardiography is a transthoracic interpretation of the electrical activity of the heart over a period of time, as detected by electrodes attached to the surface of the skin and recorded by an electrocardiogram [68]. The electrical activity of the heart is sensitive to the changes of a range of bodily functions, such as effects of the autonomic nervous system, metabolism and hormonal influences (Table 1).

\section{Measurement procedure}

Instruments are placed in a classroom, where the students can come in groups of 3 . The measurement will take approximately $1 \mathrm{~h} /$ student. At first there will be the basal or resting measurement. After that students will be presented two stress inducing tasks. The first task is a mathematical problem (cognitive stress) and the second task is a small speech given to the researcher, research assistants and others students (social stress). Speech task is divided to three parts, so each student has the opportunity to give their speech on a novel subject, while others listen.

\section{Teachers}

The teacher rated secondary outcome measures include experienced psychological strengths and difficulties measured by Strengths and Difficulties Teacher Form [58], and classroom social environment measured by Classroom Environment Scale [69]. In addition to these, in 6 months' follow-up teachers were asked to assess the pedagogical and beneficial elements of the intervention and control programs both to their students, as well as their own work load and work satisfaction (Table 1).

\section{Parents}

Parents were asked background information regarding their education, sufficiency of their salary to necessary expenses, athmosphere at home, major life changes (of their child attending the study or the whole family) and experienced psychological strengths and difficulties measured by Strengths and Difficulties Parent Form [58]. Apart from the background information, a description of all measures used in the data collection is reported in Table 1.

\section{Long run follow- up}

In addition to 6 and 12 months follow-up, a linkage to main health, or health related, registers will be done (The Social Insurance Institution of Finland; National Institute for Health and Welfare; Statistics Finland).

\section{Intervention}

A 9-week mindfulness intervention program .b (Stop \& Breathe) [46] is designed to teens aged $11-18$ years by experienced classroom teachers and mindfulness practitioners with researchers from the Oxford, Cambridge and Exeter universities. The program consists of nine 45-min group sessions and mindfulness home practices designed to improve emotional awareness, sustained attention, and attentional and emotional regulation. The program is standardized, highly recognized; and the preliminary research, though based on small intervention populations, suggests that it is effective [49]. 


\section{Active control intervention}

The control group receives a standardized relaxation program called "Relax" developed in co-operation with Folkhälsan Förbundet (based on program called "Chilla"). Relax-program aims to produce relaxation skills and holistic wellbeing for the control group attendants. Every lecture is divided in two parts, relaxation exercises and group discussion about different topics, e.g., stress, relaxation, upsides and downsides of smartphones, sleep, excercising, food and attitudes. Relaxation includes progressive muscle relaxation, a breathing excercise, visualization, choose your emotion for rest of the day and short brake for regaining energy. The dose of the program is the same as in the .b intervention, i.e. nine $45 \mathrm{~min}$ group sessions and home practices.

\section{Non-treatment control intervention}

The third arm, non-treatment-group will fill-in the same research questionnaires during the same time periods as the intervention and control groups (except the short questionnaire after the 5th lesson) in spring 2015 and in spring 2016 (ongoing). The non-treatment group will receive a shorter well-being course after the one year follow-up has been conducted.

\section{Pilot intervention study}

The acceptability and feasibility of the program has been ensured in a previous controlled pilot intervention study in two schools (4 classrooms with 19-22 students each, altogether 82 participants). The study was conducted in autumn 2012 and it indicated suitability and fit of the program to the Finnish educational system, students and staff. A qualitative assessment and the quantitative calculations showed promising effects on pupils' executive skills and well-being. Quantitative analysis showed differences between genders; among girls the greatest benefits were seen in improved self-esteem $(p=0.008)$ and stress resilience $(p=0.014)$, whereas among boys in improved self-awareness $(p=0.006)$.

\section{Treatment fidelity}

The program is delivered by 14 educated facilitators. All facilitators were provided with a self-monitoring sheet which are used for the self-assessment of their performance (e.g. intention, attitude, ability to be mindful and conduct the lesson with empathy and kindness) as well as to guarantee that the core elements of each lesson are delivered. The facilitators also assess the student's behaviour and ability to receive and internalize the core elements of a given .b lesson, as well as the teachers' presence at lessons and attitudes toward the program.

Before the intervention data collection was launched, each facilitator conducted a randomly selected .b lesson that was assessed both quantitatively and qualitatively by research group members and collagues who have attended a mindfulness-based stress reduction course but who are not part of the present research group. These lessons were also videotaped, as well as the mentioned assessment discussion. This procedure was conducted to guarantee that all facilitators are conducting "the same program with the same intention". Out of the 14 facilitators, all nine intervention group facilatators have attended a 8-week mindfulness-based stress reduction course, are educated in delivering .b school program, and practice mindfulness in their own lives. All facilitators, including active control group facilitators, except one, have received their basic education either in education or health and welfare, consisting of teachers (5), psychologists (2), health professionals (5), nutritionist (1), and a lawyer (1).

\section{Sample size}

The sample size was estimated to detect the mean difference of 0.2 standard deviation units (effect size $=0.2$ ) on main outcomes of risk for depression (RBDI), social/emotional/behavioural skills (SDQ) and resilience (RS14) between intervention and control groups with $80 \%$ power and the two-tailed $5 \%$ level of significance. The clustering of outcomes within schools was taken into account, assuming an intra-cluster (intra-school) correlation coefficient of 0.03 and assumed that on average 60 children in each school will complete the study. The required sample size was estimated to be 1090 children per group, and allowing for about $10 \%$ dropout rate, the study requires 1200 children per group and total of 2400 children to be recruited. On the RBDI and SDQ total difficulties score, an effect size of 0.2 corresponds to a mean decrease of 0.8 score on the RBDI scale and a mean decrease of 1.0 score on the SDQ scale, assuming the standard deviations of 4 for RBDI [62] and 5 for SDQ [61]. The effect size of 0.2 corresponds to a mean increase around 2.5 score on the resilience scale, assuming the standard deviation of 13 [70].

In addition to comparing the intervention and control groups, we were interested in comparing the intervention and non-treatment groups in order to gain even more strength into the study design. However, this was not our primary intrest. Since the previous research has shown also standard relaxation programs to have beneficial effects on well-being, we are expecting to find greater differences between intervention and non-treatment groups compared to intervention and control groups. Using the same assumptions to detect the mean difference of 0.3 standard deviation units (effect size of 0.3 ) between intervention and non-treatment group, the required sample size was estimated to be 486 children per group, and allowing for about $10 \%$ drop-out rate, the study requires 540 children in the non-treatment group. 


\section{Material management}

Questionnaires are stored in a locked-up room and closet at the Folkhälsan Research Center. Data is transferred into a digital format and analyzed anonymously using an identification number given for each participant, not allowing for personal identification, and is managed by designated, trained personnel. Only selected members of the research group have access to the data.

\section{Analysis plan}

Data will be analyzed on an intention to treat basis including all randomized classes in the groups to which they were randomly assigned. Descriptive statistics (mean, median or percentages as appropriate) will be used to summarize the baseline characteristics and outcomes in each group.

Statistical analysis will be done with multilevel (hierarcial) models to account for the clustering within schools. Continuous outcomes will be analysed with linear mixed effects models and categorical outcomes with generalized linear mixed effects models. Maximum likelihood estimation will be used to get unbiased and efficient parameter estimates for data with missing values in the follow-up measurements.

The effectiveness of the mindfulness intervention on primary and secondary outcomes will be first analyzed using unadjusted analyses and then adjusted for age, sex and baseline values of the outcomes. The modifying effect of factors (i.e. sex, childen's age, health status, circumstances at home, social relationships, hobbies, school achievement) on the effectiveness of mindfulness will be analysed using tests of interactions. Interaction analyses are exploratory in nature. The differences in the continuous outcomes between groups will be presented using mean differences with $95 \%$ confidence intervals. Results are expressed using odd ratios with $95 \%$ confidence intervals for categorical outcomes. Two-sided statistical tests with a $5 \%$ level of significance will be used.

\section{Discussion}

This paper describes the rationale and design of a cluster randomized controlled trial of a mindfulness intervention program among children and adolescents compared to an active control group receiving standard relaxation program, and a non-treatment group. The trial presented in this protocol aims to expand our knowledge on the effectiveness of mindfulness on a variety of behavioral, emotional, cognitive, and psychophysiological outcomes, compared to an alternative treatment and no treatment at all.

By testing the effectiveness of two alternative strategies for promoting human resilience and well-being, the present research will eventually offer new insight into the comparative usefulness of mindfulness interventions. We also focus on the unresolved questions of the mindfulness research by using a systematic and sound design to avoid methodological shortcomings.

To our knowledge, the present study is among the first ones to conduct systematic, methodologically rigorous comparative randomized research among school-aged children, on the effects of mindfulness on mental wellbeing.

\section{Abbreviations}

No abbreviations used.

\section{Acknowledgements}

The research team would like to thank all the members of the scientific advisory board for their valuable contribution to the Healthy Learning Mind study. We owe special compliments to Martina Rosenqvist, Ritva Linden and Jenny Penna for their valuable contribution to the research project as the coordinators for the research project. We are very grateful for the co-operation with Folkhälsan förbundet and especially to the director Viveca Hagmark, as well as to Erika Fogelberg and Mikaela Wiik. Also Samu Sundqvist, Sari Markkanen and Anna-Maria Majava earn a big thank you for their effort in creating the Relax control program. Additionally we would like to thank Eva Roos for her advices regarding the study design, and Janne Pitkäniemi and Jari Haukka for the statistical expertice. Furthermore, the research team is also grateful to all the participating schools, their principals and teachers, the children and adolescents and their parents and all the assistants who participated in the data collection.

\section{Funding}

This project is funded by Signe and Ane Gyllenberg Foundation; Juho Vainio Foundation; Mats Brommels Foundation; Yrjö Jahnsson Foundation; Ministry of Social Affairs and Health. The study protocol has undergone peer-review by all the funding bodies.

\section{Availability of data and material}

Data is available from the authors on request.

\section{Authors' contributions}

All authors contributed to the design and content of the study protocol. More specifically, S-MV conceived the study, S-MV, ML, NH, MH, AR, SM, NS, and SS were in charge of the study design, $A B$ and TV were in charge of the statistical expertise, ML, MH, PS, AR and SM were in charge of the psychological and child/adolescent psychiatric expertise, S-MV, ML, MH, NH, SM, NS and SS were in charge of the data collection procedure, S-MV, NS, NH, PS and SS were in charge of the epidemiological expertise, S-MV, ML, NH, MH, NS, PS, AR, SM were in charge of the measures, S-MV drafted the manuscript. All authors contributed to the refinement of the study protocol, and have read and approved the final manuscript.

\section{Competing interests}

The authors declare that they have no competing interests.

\section{Consent for publication}

Not applicable.

\section{Ethics approval and consent to participate}

The study protocol has been approved in the ethical review board of the University of Helsinki (in humanities and social and behavioural sciences), Statement 1/2014. The study protocol has also been reviewed and approved in the educational departments of the respective school districts. A written informed consent is requested from all participants and their parents. The linkage of the survey data to national health registries will be carried out based on appropriate authority and participant consent.

The participants were informed that the participation in the study is voluntary and that they may withdraw from the study at any time without giving a reason. Also the teachers received their letter of invitation where information regarding e.g. the questionnaires and difficult feelings that some questions may raise in some children was presented. The teachers were at the classrooms while participants filled in the questionnaires and during the intervention and control treatment sessions. Parents filled in their 
questionnaire at home, and brought it in a closed envelope to school from where researchers collect them as well as teachers' questionnaires.

\section{Sponsor}

Folkhälsan Research Center/University of Helsinki (Department of Public Health).

\section{Scientific advisory board of the study}

Professor Raimo Lappalainen, University of Jyväskylä, raimo.lappalainen@jyu.fi PhD Päivi Lappalainen, University of Jyväskylä, paivi.k.lappalainen@jyu.fi Adjunct professor Mirjam Kalland, University of Helsinki, mirjam.kalland@helsinki.fi

Adjunct professor Nelli Hankonen, University of Tampere, nelli.hankonen@staff.uta.fi

Adjunct professor Päivi Santalahti, Institute for Health and Welfare, paivi.santalahti@thl.fi

\section{Author details \\ ${ }^{1}$ Folkhälsan Research Center, Topeliuksenkatu 20, 00250 Helsinki, Finland. ${ }^{2}$ Department of Public Health, University of Helsinki, Helsinki, Finland. ${ }^{3}$ Institute of Behavioural Sciences, University of Helsinki, Helsinki, Finland. ${ }^{4}$ School of Social Sciences and Humanities, University of Tampere, Tampere, Finland. ${ }^{5}$ National Institute for Health and Welfare, Helsinki, Finland. ${ }^{6}$ Unit of Psychology, University of Oulu, Oulu, Finland. ${ }^{7}$ Department of Adolescent Psychiatry, Helsinki University Central Hospital, Helsinki, Finland. ${ }^{8}$ Department of Mental Health and Substance Abuse Services, National Institute for Health and Welfare, Helsinki, Finland. ${ }^{9}$ Department of Teacher Education, University of Helsinki, Helsinki, Finland. ${ }^{10}$ Department of Biostatistics, University of Turku, Turku, Finland. ${ }^{11}$ Department of Public Health, University of Skövde, Skövde, Sweden. ${ }^{12}$ Department of Public Health, University of Turku, Turku, Finland.}

Received: 22 May 2016 Accepted: 1 July 2016

Published online: 11 July 2016

\section{References}

1. Salzman A, Goldin P. Mindfulness-based stress reduction for school-age children. New Harbinger: Context Press; 2008.

2. Meiklejohn J, Phillips C, Lee Freedman M, Lee Griffin M, Biegel G, Roach A, et al. Integrating mindfulness training into K-12 education: fostering the resilience of teachers and students. Mindfulness. 2012;3(4):291-307.

3. Merikangas KR, Nakamura EF, Kessler RC. Epidemiology of mental disorders in children and adolescents. Dialogues Clin Neurosci. 2009;11(1):7-20.

4. Kinnunen P, Laukkanen E, Kiviniemi V, Kylma J. Associations between the coping self in adolescence and mental health in early adulthood. J Child Adolesc Psychiatr Nurs. 2010;23(2):111-17

5. Patel V, Flisher AJ, Hetrick S, McGorry P. Mental health of young people: a global public-health challenge. Lancet. 2007;369:1302-13.

6. Willard W, Long A, Phipps S. Life stress versus traumatic stress: the impact of life events on psychological functioning in children with and without serious illness. Psycho Trauma. 2016;8(1):63-71. doi:10.1037/tra0000017.

7. Kovacs D, Eszlari N, Petschner P, Pap D, Vas S, Kovacs P, et al. Interleukin-6 promoter polymorphism interacts with pain and life stress influencing depression phenotypes. J neural transm (Vienna). 2016;123(5):541-8. doi:10. 1007/s00702-016-1506-9.

8. Mundy EA, Weber M, Rauch SL, Killogore WD, Simon NM, Pollack MH, et al. Adult anxiety disorders in relation to trait anxiety and perceived stress in childhood. Psychol Rep. 2015;117(2):473-89. doi:10.2466/02.10. PRO.117c17z6

9. Burke CA. Mindfulness-based approaches with children and adolescents: a preliminary review of current research in an emergent field. J Child Fam Stud. 2010;19(2):133-44.

10. Kabat-Zinn J. An out-patient program in behavioral medicine for chronic pain patients based on the practice on mindfulness meditation: theoretica considerations and preliminary results. Gen Hosp Psychiatry. 1982:4:33-47.

11. Kabat-Zinn J. Mindfulness-based stress reduction (MBSR). Constructivism Hum Sci. 2003:8(2):73-107.

12. Segal ZV, Williams JMG, Teasdale JD. Mindfulness-based cognitive therapy for depression: a New approach to preventing relapse. New York: Guilford Press; 2002.

13. Linehan MM. Cognitive behavioral treatment of borderline personality disorder. New York, NY: Guilford Press; 1993.
14. Hayes SC, Strosahl KD, Wilson KG. Acceptance and commitment therapy: an experiential approach to behavior change. New York: Guilford Press; 1999. p. 304. xvi.

15. Hayes SC, Wilson KG. Mindfulness: method and process. Clin Psychol Sci Pract. 2003;10(2):161-5

16. Blackledge J. An introduction to relational frame theory: basics and applications. The Behavior Analyst Today. 2003;3:421-33.

17. Gotink RA, Chu P, Busschbach JJ, Benson H, Fricchione GL, Hunink MG. Standardised mindfulness-based interventions in healthcare: an overview of systematic reviews and meta-analyses of RCTs. PLoS One. 2015;16;10(4): e0124344. doi:10.1371/journal.pone.0124344.

18. Hofmann SG, Sawyer AT, Witt AA, Oh D. The effect of mindfulness-based therapy on anxiety and depression: a meta-analytic review. J Consult Clin Psychol. 2010;78(2):169-83

19. Chiesa A, Calati R, Serretti A. Does mindfulness training improve cognitive abilities? A systematic review of neuropsychological findings. Clin Psychol Rev. 2011:31(3):449-64

20. Barnhofer T, Crane C, Hargus E, Amarasinghe M, Winder R, Williams JM. Mindfulness-based cognitive therapy as a treatment for chronic depression: a preliminary study. Behav Res Ther. 2009;47(5):366-73. doi:10.1016/j.brat. 2009.01.019.

21. Faude-Lang V, Hartman M, Schmidt EM, Humpert P, Nawroth P, Herzof W. Acceptance - and mindfulness - based group intervention in advanced type 2 diabetes patients: therapeutic concept and practical experiences. Psychoter Psychosom Med Psychol. 2010;60(5):185-89.

22. Rosenzweig S, Reibel DK, Greeson JM, Edman JS, Jasser SA, McMearty KD, et al. Mindfulness-based stress reduction is associated with improved glycemic control in type 2 diabetes mellitus: a pilot study. Altern Ther Health Med. 2007;13(5):36-8

23. Zylowska L, Ackerman DL, Yang MH, Futrell JL, Horton NL, Hale TS, et al. Mindfulness meditation training in adults and adolescents with ADHD: a feasibility study. J Atten Disord. 2008;11(6):737-46.

24. Smalley SL, Loo SK, Hale TS, Shrestha A, McGough J, Flook L, et al. Mindfulness and attention deficit hyperactivity disorder. J Clin Psychol. 2009;65(10):1087-98.

25. Brewer JA, Mallik S, Babuscio TA, Nich C, Johnson HE, Deleone CM, et al. Mindfulness training for smoking cessation: results from a randomized controlled trial. Drug Alcohol Depend. 2011;119(1-2):72-80.

26. Vidrine Jl, Businelle MS, Cinciripini P, Li Y, Marcus MT, Waters AJ, et al. Associations of mindfulness with nicotine dependence, withdrawal, and agency. Subst Abus. 2009;30(4):318-27. doi:10.1080/08897070903252973.

27. Godfrey KM, Gallo LC, Afari N. Mindfulness-based interventions for binge eating: a systematic review and meta-analysis. J Behav Med. 2015;38(2):348-62. doi:10.1007/s10865-014-9610-5.

28. Brewer JA, Sinha R, Chen JA, Michalsen RN, Babuscio TA, Nich C, et al. Mindfulness training and stress reactivity in substance abuse: results from a randomized, controlled stage I pilot study. Subst Abus. 2009;30(4):306-17.

29. Chiesa A, Serretti A. Mindfulness-based stress reduction for stress management in healthy people: a review and meta-analysis. J Altern Complement Med. 2009;15(5):593-600.

30. Howell AJ, Digdon NL, Buro K. Mindfulness predicts sleep-related self-regulation and well-being. Personal Individ Differ. 2010;48:419-24. doi:10.1016/j.paid.2009. 11.009

31. Brown K, Ryan R. The benefits of being present: mindfulness and its role in psychological well-being. J Pers Soc Psychol. 2003:84(4):822-48.

32. Hölzel BK, Carmody J, Vangel M, Congleton C, Yerramsetti SM, Gard T, et al. Mindfulness practice leads to increases in regional brain gray matter density. Psychiatry Res Neuroimaging. 2011a;191(1):36-43.

33. Hölzel BK, Lazar SW, Gard T, SchumanOlivier Z, Vago DR, Ott U. How does mindfulness meditation work? proposing mechanisms of action from a conceptual and neural perspective. Perspect Psychol Sci. $2011 \mathrm{~b}$; 6(6):537-59.

34. Chiesa A, Brambilla P, Serretti A. Neuro-imaging of mindfulness meditations: implications for clinical practice. Epidemiol Psychiatr Sci. 2011;20(2):205-10.

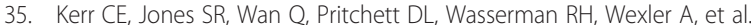
Effects of mindfulness meditation training on anticipatory alpha modulation in primary somatosensory cortex. Brain Res Bull. 2011;85(3-4):96-103.

36. Warren Brown K, Goodman RJ, Inzlicht M. Dispositional mindfulness and the attenuation of neural responses to emotional stimuli. SCAN. 2013:8:93-9. doi:10.1093/scan/nss004 
37. Lutz J, Herwig U, Opialla S, Hittmeyer A, Jäncke L, Rufer M, et al. Mindfulness and emotion regulation —an fMRI study. Soc Cogn Affect Neurosci. 2014;9(6):776-85.

38. Singh NN, Lancioni GE, Joy SDS, Winton ASW, Sabaawi M, Wahler RG, et al. Adolescents with conduct disorder can be mindful of their aggressive behavior. J Emot Behav Disord. 2007;15(1):56-63.

39. Bogels S, Hoogstad B, van Dun L, de Schutter S, Restifo K. Mindfulness training for adolescents with externalizing disorders and their parents. Behav Cogn Psychother. 2008;36(2):193-209.

40. Biegel G, Brown K, Shapiro S, Schubert C. Mindfulness-based stress reduction for the treatment of adolescent psychiatric outpatients: $A$ randomized clinical trial. J Consult Clin Psychol. 2009;77(5):855-66.

41. Van der Oord S, Bögels S, Peijnenburg D. The effectiveness of mindfulness training for children with ADHD and mindful parenting for their parents. J Child Fam Stud. 2012;2:139-47.

42. Weijer-Bergsma E, Forsma A, Bruin E, Bögels S. The effectiveness of minfulness training on behavioral problems and attentional functioning in adolescents with ADHD. J Child Fam Stud. 2012;5:775-87.

43. Napoli M, Krech PR, Holley LC. Mindfulness training for elementary school students: the attention academy. J Appl Sch Psychol. 2005;21(1):99-125.

44. Flook L, Smalley SL, Kitil MJ, Galla BM, Locke J, Ishijima E, et al. Effects of mindful awareness practices on executive functions in elementary school children. J Appl Sch Psychol. 2010;26(1):70-95.

45. Biegel G, Brown K. Assessing the efficacy of an adapted in-class mindfulness-based training program for school-age children: a pilot study. In A Research Brief for Mindful Schools. 2011. www.mindfulschools.org/pdf/ Mindful\%20Schools\%20Pilot\%20Study\%20Whitepaper.pdf.

46. Huppert FA, Johnson DM. A controlled trial of mindfulness training in schools: the importance of practice for an impact on well-being. J Posit Psychol. 2010;5(4):264-74.

47. Joyce A, Etty-Leal J, Zazryn T, Hamilton A. Exploring mindfulness meditation program on the mental health of upper primary children - a pilot study. Adv School Ment Health Promot. 2010;3:17-25.

48. Klatt M, Harpster K, Browne E, White S, Case-Smith J. Feasibility and preliminary outcomes for move-into-learning: an arts-based mindfulness classroom intervention. J Positive Psychol. 2013;8(3):233-41.

49. Kuyken W, Weare K, Ukoumunne O, Vicary R, Motton N, Burnett R, et al. Effectiveness of the mindfulness in schools programme: non-randomised controlled feasibility study. Br J Psychiatry. 2013;203(2):126-31.

50. Jennings PA, Frank JL, Snowberg KE, Coccia MA, Greenberg MT. Improving classroom learning environments by Cultivating Awareness and Resilience in Education (CARE): results of a randomized controlled trial. Sch Psychol Q. 2013. doi:10.1037/spq0000035.

51. Kallapiran K, Koo S, Kirubakaran R, Hancock K. Review: Effectiveness of mindfulness in improving mental health symptoms of children and adolescents: a meta-analysis. Child Adolesc Mental Health. 2015;20(4):182-94. doi:10.1111/camh.12113.

52. Zoogman S, Goldberg SB, Hoyt WT, Miller L. Mindfulness interventions with youth: a meta-analysis. Mindfulness. 2014;6:290-302. doi:10.1007/s12671013-0260-4.

53. Broderick PC, Jennings PA. Mindfulness for adolescents: a promising approach to supporting emotion regulation and preventing risky behavior. N Dir Youth Dev. 2012:136:111-26.

54. Black DS, Fernando R. Mindfulness training and classroom behavior among lower-income and ethnic minority elementary school children. J Child Fam Stud. 2014;23(7):1242-46

55. Schulz KF, Altman DG, Moher D. CONSORT 2010 statement: updated guidelines for reporting parallel group randomised trials. J Pharmacol Pharmacother. 2010;1(2):100-7. doi:10.4103/0976-500X.72352.

56. Campbell MK, Piaggio G, Elbourne DR, Altman DG. Consort 2010 statement: extension to cluster randomised trials. BMJ. 2012;345:e5661. doi:10.1136/bmj. e5661.

57. Raitasalo R. Mielialakysely. Suomen oloihin Beckin lyhyen depressiokyselyn pohjalta kehitetty masennusoireilun ja itsetunnon kysely. Kela, Sosiaali- ja terveysturvan tutkimuksia, 86, 2007. Helsinki; 2007.

58. Goodman R. The strengths and difficulties questionnaire: a research note. J Child Psychol Psychiatry. 1997;38:581-86.

59. Wagnild GM, Young HM. Development and psychometric evaluation of the resilience scale. J Nurs Meas. 1993;1:165-78.

60. Losoi H, Turunen S, Wäljas M, Helminen M, Öhman J, Julkunen J, et al. Psychometric Properties of the Finnish Version of the Resilience Scale and its Short Version. Psychology, Community \& Health. North America. 2013; 2(1):1-10.

61. Koskelainen M, Sourander A, Kaljonen A. The strengths and difficulties questionnaire among finnish school-aged children and adolescents. Eur Child Adolesc Psychiatry. 2000;9:277-84.

62. Kaltiala-Heino R, Rimpelä M, Rantanen P, Laippala P. Finnish modification of the 13-item Beck DepressionInventory in screening an adolescent population for depressiveness and positive mood. Nordic J Psychiatry. 1999; 53:451-57.

63. Konu A, Alanen E, Lintonen T, Rimpelä M. Factor structure of the school well-being model. Health Educ Res. 2002a;17:732-42.

64. Korkman M, Kirk U, Kemp S. Psychological Corporation. NEPSY-II. secondth ed. 2007.

65. Wechsler D. The Wechsler intelligence scale for children-third edition. San Antonio: The Psychological Corporation; 1991.

66. Delis D, Kaplan E, Kramer J. The delis-kaplan executive function system. San Antonio: Psychological Corporation; 2001.

67. Schell A, Dawson M, Filion D. Psychophysiological correlates of electrodermal lability. Psychophysiology. 1988;25(6):619-32.

68. Becker D. Fundamentals of electrocardiography interpretation. Anesth Prog. 2006;53(2):53-64.

69. Moos RH, Trickett E. Classroom environment scale manual: second edition. Palo Alto: Consulting Psychologists Press; 1987.

70. Pritzker S, Minter A. Measuring adolescent resilience: an examination of the cross-ethnic validity of the RS-14. Child Youth Serv Rev. 2014;44:328-33.

\section{Submit your next manuscript to BioMed Central and we will help you at every step:}

- We accept pre-submission inquiries

- Our selector tool helps you to find the most relevant journal

- We provide round the clock customer support

- Convenient online submission

- Thorough peer review

- Inclusion in PubMed and all major indexing services

- Maximum visibility for your research

Submit your manuscript at www.biomedcentral.com/submit
Biomed Central 